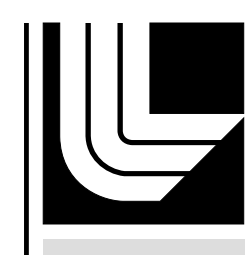

LAWRENCE LIVERMORE N A T IO N A L LABORATORY

\title{
On The Electronic Configuration in $\mathrm{Pu}$
}

J. G. Tobin, P. Soderlind, A. Landa, K. T. Moore, A. J. Schwartz, B. W. Chung, M. A. Wall, J. M. Wills, R. G. Haire, A. L. Kutepov

February 6, 2007

MRS 2006 Fall Meeting Proceedings: Symposium OO: Actinides -- Basic Science, Applications, and Technology 
This document was prepared as an account of work sponsored by an agency of the United States Government. Neither the United States Government nor the University of California nor any of their employees, makes any warranty, express or implied, or assumes any legal liability or responsibility for the accuracy, completeness, or usefulness of any information, apparatus, product, or process disclosed, or represents that its use would not infringe privately owned rights. Reference herein to any specific commercial product, process, or service by trade name, trademark, manufacturer, or otherwise, does not necessarily constitute or imply its endorsement, recommendation, or favoring by the United States Government or the University of California. The views and opinions of authors expressed herein do not necessarily state or reflect those of the United States Government or the University of California, and shall not be used for advertising or product endorsement purposes. 


\title{
MRS Proceedings: On the electronic configuration in $\mathrm{Pu}$
}

\author{
J.G. Tobin ${ }^{1}$, P. Söderlind ${ }^{1}$, A. Landa ${ }^{1}$, K.T. Moore ${ }^{1}$, A.J. Schwartz ${ }^{1}$, B.W. Chung ${ }^{1}$, \\ M.A. Wall ${ }^{1}$, J.M. Wills ${ }^{2}$, R.G. Haire ${ }^{3}$, and A.L. Kutepov ${ }^{4}$ \\ 1.Lawrence Livermore National Laboratory, Livermore, CA 94550, USA \\ 2.Los Alamos National Laboratory, Los Alamos, NM 87545, USA \\ 3.Oak Ridge National Laboratory, Oak Ridge, TN, USA \\ 4.Russian Federation Nuclear Center, Inst. of Technical Physics (VNIITF), Snezhinsk, Russia
}

\begin{abstract}
Synchrotron-radiation-based x-ray absorption, electron energy-loss spectroscopy, and densityfunctional calculations have been used to study the electron configuration in Pu. These methods suggest a $5 f^{n}$ configuration for $\mathrm{Pu}$ of $5 \leq n<6$, with $n \neq 6$.
\end{abstract}

\section{X-ray Absorption and Electron Energy Loss Spectroscopy}

The data from x-ray absorption (XAS) and electron energy loss spectroscopy (EELS) indicate that the number of $5 f$ electrons, $n$, involved in bonding of Pu must be at least as great as 5 and less than 6 . The equivalence of the XAS and high-energy EELS measurements for assessing the electronic states has already been demonstrated for $\mathrm{Ce}$ and $\mathrm{Pu}$, as well as for other actinides [15]. The argument against $n=6$ will now be discussed in a step-wise fashion.

I. The relative diminishment of the $\mathrm{Pu} 4 d_{3 / 2}$ peaks indicates strong relativistic effects in the Pu $5 f$ states, i.e., a jj-coupling or jj-skewed intermediate coupling scheme.

As can be seen in Figure 1, the intensity of the $4 d_{3 / 2}$ peak of Pu is significantly reduced versus that for $\mathrm{U}$ [6]. This large reduction is driven by the electric dipole selection rule that forbids the transition from a pure $d_{3 / 2}$ peak into a pure $f_{7 / 2}$ peak. This reduction also implies that the $\mathrm{Pu} 5 f$ states must be split into two lobes, the lower (mainly occupied) lobe being principally pure $5 / 2$ character and the upper (unoccupied) lobe being principally pure $7 / 2$ character. This picture is shown schematically in Figure 2. This result is independent of any particular theoretical model for spin-orbit splitting or the calculation of x-ray absorption cross section and thus does not depend upon the details of the branching ratio analysis presented previously [1,4].

II. Coupled with the result in I above, the absence of a pre-peak in the Pu 5d XAS and EELS indicates that $\boldsymbol{n}$ must be at least 5 .

As shown in Figure 3, there is a pre-peak in the EELS $5 d$ to $5 f$ transition for Th and $\mathrm{U}$, but not for Pu. Similar results (Figure 4) have been obtained for $5 d$ XAS. [2]. The pre-peak structure in the $4 d$ XAS of the rare earths was explained many years ago by Dehmer et al. [7]: it is driven by the combination of angular momentum coupling between the $4 d$ and $4 f$ states and the dependence of the Coulombic energy term upon the coupling details. The spectroscopic transition for the rare earths can be summarized as follows.

$4 d^{10} 4 f^{\mathrm{n}}+\mathrm{h} v \rightarrow 4 d^{9} 4 f^{\mathrm{n}+1}$

Eq A 
When $n=14$, there is no transition. When $n=13$, there is a single main peak, without any prepeaks [2]. This is because partial occupation of both the $d$ and $f$ states is required for coupling. If $n=13$ and $n+1=14$, then in the final state the $4 f$ level is filled and no coupling occurs. For the lighter actinides $(n<6)$, the situation is similar but not identical.

$5 d_{5 / 2}$, pure $\mathrm{jj}, n<6$

$\left(5 d_{3 / 2}\right)^{4}\left(5 d_{5 / 2}\right)^{6}\left(5 f_{5 / 2}\right)^{n}\left(5 f_{7 / 2}\right)^{0}+\mathrm{hv} \rightarrow\left(5 d_{3 / 2}\right)^{4}\left(5 d_{5 / 2}\right)^{5}\left(5 f_{5 / 2}\right)^{n+1}\left(5 f_{7 / 2}\right)^{0}+\left(5 d_{3 / 2}\right)^{4}\left(5 d_{5 / 2}\right)^{5}\left(5 f_{5 / 2}\right)^{\mathrm{n}}\left(5 f_{7 / 2}\right)^{1}$

Eq B1

Eq B2

$5 d_{3 / 2}$, pure $\mathrm{jj}, n<6$

$\left(5 d_{3 / 2}\right)^{4}\left(5 d_{5 / 2}\right)^{6}\left(5 f_{5 / 2}\right)^{\mathrm{n}}\left(5 f_{7 / 2}\right)^{0}+\mathrm{hv} \rightarrow\left(5 d_{3 / 2}\right)^{3}\left(5 d_{5 / 2}\right)^{6}\left(5 f_{5 / 2}\right)^{n+1}\left(5 f_{7 / 2}\right)^{0}$

Again transitions from $d_{3 / 2}$ to $f_{7 / 2}$ are forbidden. For the pre-peaks at lower hv, we need only consider the $\left(5 f_{5 / 2}\right)^{n+1}$ terms. (Transitions into the $f_{7 / 2}$ states will generally need higher energies and pre-peaks that might be associated with this transition will be coincident with the main $f_{5 / 2}$ spectral structure and lost therein.) The final state $5 / 2$ level will be filled at $n+1=6$ or $n=5$. For $n<5$ (e.g., Th and $\mathrm{U}$ ), there will be pre-peaks. The absence of pre-peaks for $\mathrm{Pu}$, both the $\alpha$ and the $\delta$ phase, indicates that $n$ must be 5 or greater. This raises the question: What about an $n$ of 6 and above for Pu? For $n \geq 6$, the transition looks like this.

$5 d_{5 / 2}$, pure jj, $n \geq 6$

$\left(5 d_{3 / 2}\right)^{4}\left(5 d_{5 / 2}\right)^{6}\left(5 f_{5 / 2}\right)^{6}\left(5 f_{7 / 2}\right)^{\mathrm{n}-6}+\mathrm{hv} \rightarrow\left(5 d_{3 / 2}\right)^{4}\left(5 d_{5 / 2}\right)^{5}\left(5 f_{5 / 2}\right)^{6}\left(5 f_{7 / 2}\right)^{\mathrm{n}-5}$

$\mathrm{Eq} \mathrm{C} 1$

$5 d_{3 / 2}$, pure $\mathrm{jj}, \mathrm{n} \geq 6$

$\left(5 d_{3 / 2}\right)^{4}\left(5 d_{5 / 2}\right)^{6}\left(5 f_{5 / 2}\right)^{6}\left(5 f_{7 / 2}\right)^{\mathrm{n}-6}+\mathrm{hv} \rightarrow \quad$ No Transition Allowed

$\mathrm{Eq} \mathrm{C} 2$

Once again, there will be no transition in Eq C2 because $d_{3 / 2}$ to $f_{7 / 2}$ transitions are forbidden.

The situation is different in another, subtle way as well. A filled $5 f_{5 / 2}$ level now stands between the partially occupied $5 d$ and $5 f_{7 / 2}$ levels. It is unclear whether angular momentum coupling (and thus pre-peak formation) will occur under these conditions. In fact, the spectrum from $\mathrm{AmH}_{2}$ shown in Figure 3 exhibits no evidence of pre-peaks. Although this is not a spectrum from an elemental actinide, past experiments have shown that for a given actinide, the peak-ratio values tend to be grouped together, with larger separations between the elemental groups [8]. The grouping tended to be tightest for actinide elements that exhibited a strong localization even in the elemental, metallic state.

\section{The presence of two edges in the Pu $5 d$ XAS and a substantial peak in the $4 d_{3 / 2}$ XAS indicate that $n<6$.}

As can be seen in the $5 d \mathrm{XAS}$ of both $\alpha$ - and $\delta$-Pu (Figure 4), there are two strong edges for $\mathrm{h} v$ $=110-120 \mathrm{eV}$. A similar, albeit weaker, structure is observed in the EELS of $\alpha-$ and $\delta$-Pu, as shown in Figure 3. These two steps are the leading edges of the main $5 d_{5 / 2}$ (about $110 \mathrm{eV}$ ) and $5 d_{3 / 2}$ (about $120 \mathrm{eV}$ ) transitions. (Confirmation of the initiation of the $5 d$ transition near $110 \mathrm{eV}$ can be gleaned from photoelectron spectroscopy. See Ref. 9 and references therein.) As illustrated in Eq C2, if $n=6$ or more, one of the two transitions is lost. This is exactly what has happened in $\mathrm{AmH}_{2}$, as shown in Figure 3. Here, the second spin-orbit split peak is essentially gone, or at least strongly attenuated, and lost in the tail of the other peak/edge. The overall peak 
structure of the "giant resonance" of $\mathrm{AmH}_{2}$ is now about half as wide as that of the $\mathrm{Pu}$. Thus, the presence of two strong edges indicates that $n<6$ for $\mathrm{Pu}$.

An analogous process will occur for the $4 d$ to $5 f$ transitions. For a pure jj coupling scheme and electric dipole selection rules, the $4 d_{3 / 2}$ peak should vanish. In reality, there will be mixing between the pure $5 f_{5 / 2}$ and $5 f_{7 / 2}$ states, so at $n=6$, one would expect a small but almost negligible $4 d_{3 / 2}$ peak. Again, this is exactly what has been observed for $\mathrm{AmH}_{2}$ as shown in Figure 5 [8]. The $\mathrm{Pu} 4 d_{3 / 2}$ peak is too large for $n=6$, and therefore for $\mathrm{Pu}, n<6$.

What about the possibilities for Am being $n>6$ ? This seems unlikely. As will be discussed below, there is very strong evidence that Am has $n=6$. Additionally, recent measurements on Cm [10] indicate that $n=7$, within an LS like coupling scheme. The return to an LS skewed intermediate case for the heavier actinides is consistent with the atomic model of van der Laan and Thole [11], which has been used to explain the cross-sectional behavior of the lighter actinides $[1,4]$.

It is useful to consider the possibility of phase specific variations of the XAS/EELS findings and thus the $n$ value for $\mathrm{Pu}$. As can be seen in Figures 3 and 4, within each technique, the results for $\alpha$ and $\delta$ are quite similar for the $5 d$ to $5 f$ transitions. Additionally, as shown in Figure 6 , the $N_{4,5}$ electron-energy loss spectra for $\alpha$ and $\delta \mathrm{Pu}$ are again similar [8]. The experimental data hence point towards the conclusion that for $\mathrm{Pu} n$ is less than 6 and greater than or equal to 5 , either for the $\alpha$ or the $\delta$ phase. This does not rule out that there may be phase specific variations between the spectra, but these are small compared to the variation from element to element.

Thus, to summarize for Pu $5 f$ occupation: From XAS and EELS, $5 \leq n<6$, with $n \neq 6$.

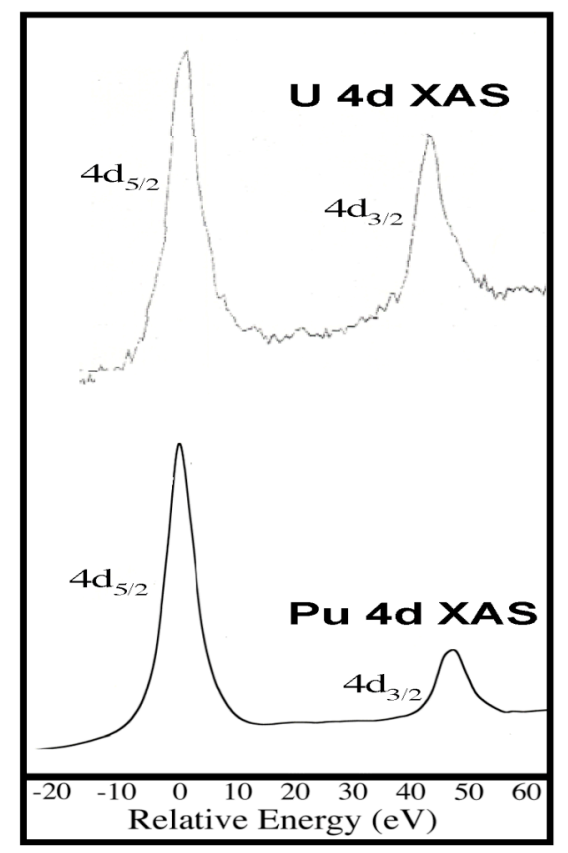

Figure 1.

The 4d to 5f XAS transition results for $\mathrm{Pu}$ [1] and $U$ [6] are shown here. The photon energies were near $800 \mathrm{eV}$. 


\section{Simple Picture derived from the spectroscopic analysis}

Result of nonmagnetic calculation, including spinorbit in the Pu 5f's
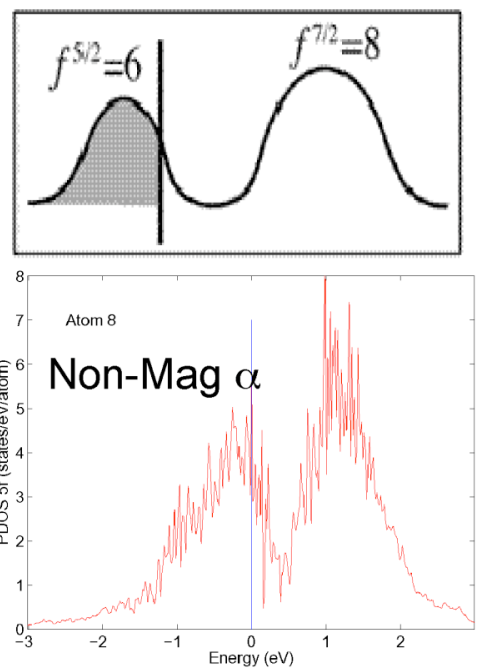

\section{Result of anti- ferromagnetic calculation, including spin- orbit in the Pu 5f's}

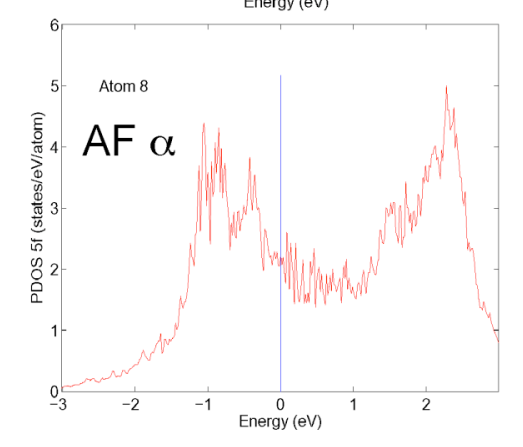

Figure 2.

The two lobed structure of the Pu $5 f$ density of states is shown here. Top: the simple picture derived from the spectroscopic analysis. Middle: the result of non-magnetic calculation by Kutepov, including the spin-orbit splitting in the $\mathrm{Pu} 5 f$ states. Bottom: the result of an anti-ferromagnetic calculation by Kutepov, including the spin-orbit splitting in the Pu $5 f$ states. See Ref. 1 for the details concerning this figure.

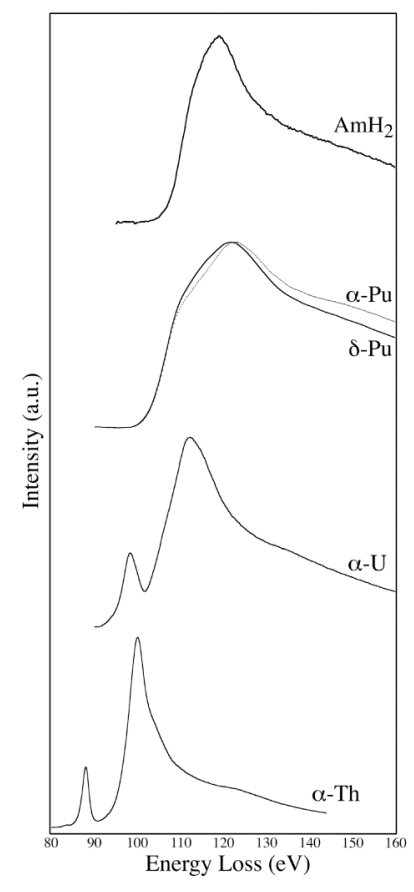

Figure 3.

The EELS spectra of the $5 d$ to $5 f$ transitions of Th (bottom), U, (second from bottom), $\mathrm{Pu}$ (second from top), and $\mathrm{AmH}_{2}$ (top) are shown here. The Th, $\mathrm{U}$, and $\mathrm{Pu}$ data are from Ref. 2. The $\mathrm{AmH}_{2}$ data are from Ref. 8. 


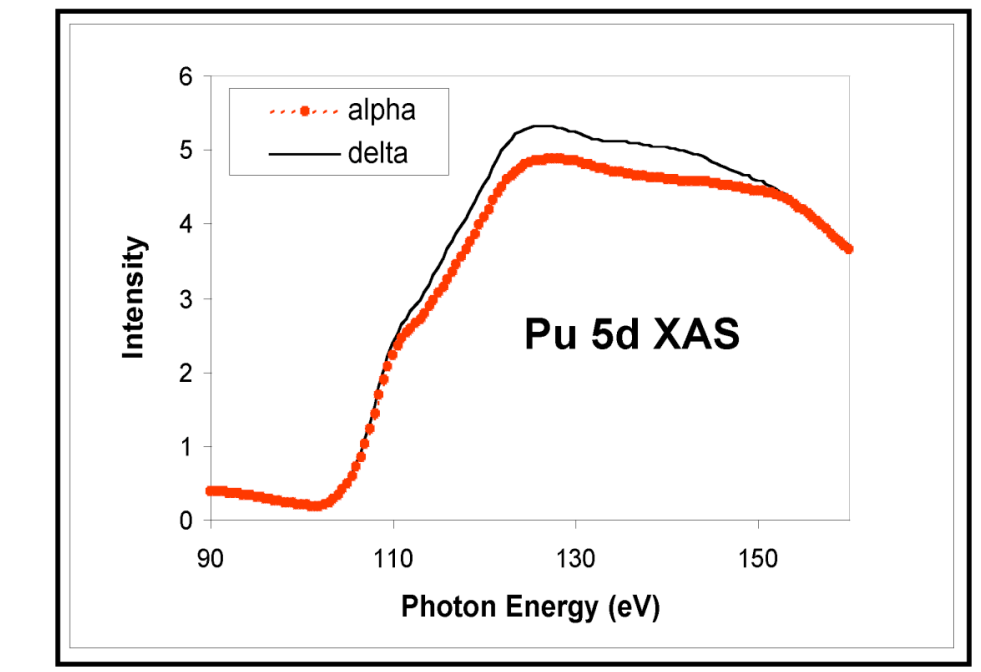

Figure 4.

The XAS data for the $5 d$ to $5 f$ transition in $\mathrm{Pu}$ is shown here. The spectra are taken from Ref. 2.

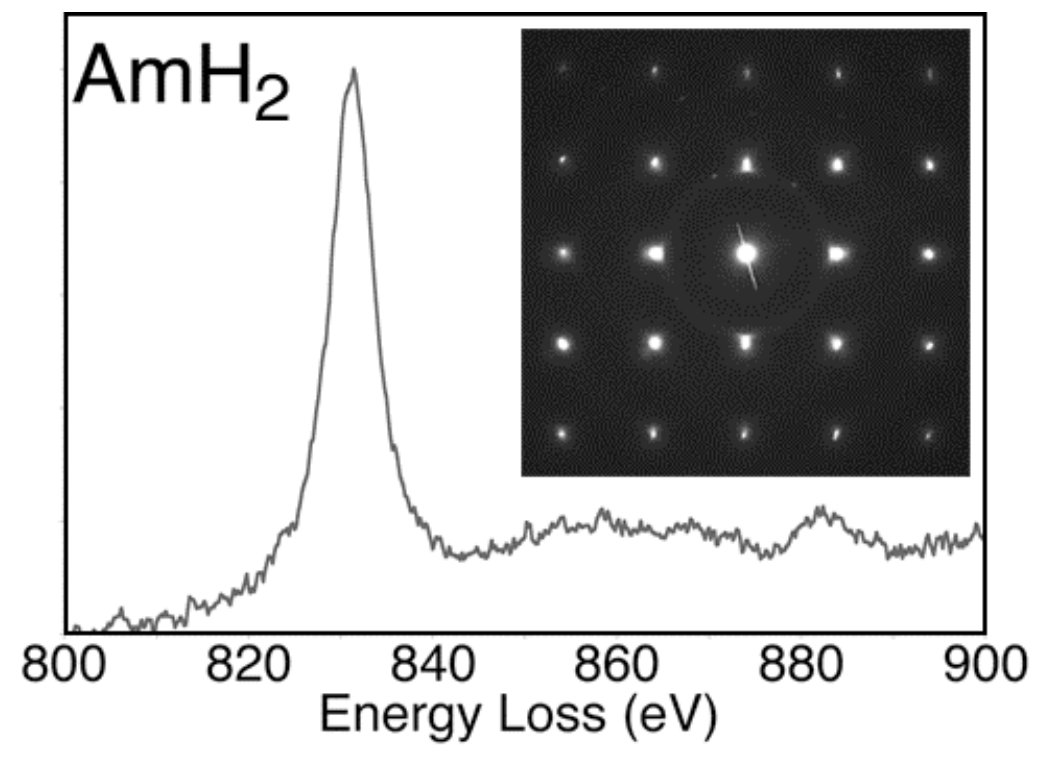

Figure 5.

The electron diffraction and EELS data for the $4 d$ to $5 f$ transition of Am, from an $\mathrm{AmH}_{2}$ sample, is shown here. The data is taken from Ref. 8.

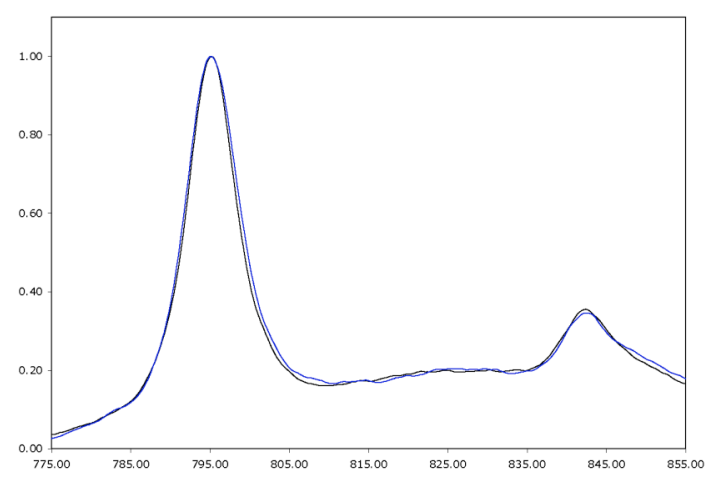

Figure 6.

The EELS data for the $4 d$ to $5 f$ transition in $\alpha$ and $\delta$ $\mathrm{Pu}$ is shown here. The spectra are taken from Ref. 8. 


\section{Acknowledgements}

We thank Dr. L.V. Pourovskii for providing us with the AMF-DMFT DOS. This work was performed under the auspices of U.S. Department of Energy by the University of California, Lawrence Livermore National Laboratory under contract No. W-7405-Eng-48. Work that was performed by JGT was supported in part by the Office of Basic Energy Science at the U.S Department of Energy. The ALS and the Spectromicroscopy Facility have been built and operated under funding from the Office of Basic Energy Science at DOE.

\section{References}

1. J.G. Tobin, K.T. Moore, B.W. Chung, M.A. Wall, A.J. Schwartz, G. van der Laan, and A.L. Kutepov, Phys. Rev. B 72, 085109 (2005).

2. K.T. Moore, M.A. Wall, A.J. Schwartz, B.W. Chung, D.K. Shuh, R.K. Schulze, and J.G. Tobin, Phys. Rev. Lett. 90, 196404 (2003) and references therein.

3. K.T. Moore, B.W. Chung, S.A. Morton, S. Lazar, F.D. Tichelaar, H.W. Zandbergen, P. Söderlind, G. van der Laan, A.J. Schwartz, and J.G. Tobin, Phys. Rev. B 69, 193104 ( 2004).

4. G. van der Laan, K.T. Moore, J.G. Tobin, B.W. Chung, M.A. Wall, and A.J. Schwartz, Phys. Rev. Lett. 93, 097401 (2004).

5. K.T. Moore, M.A. Wall, A.J. Schwartz, B.W. Chung, S.A. Morton, J.G. Tobin, S. Lazar, F.D. Tichelaar, H.W. Zandbergen, P. Söderlind, and G. van der Laan, Phil. Mag. 84, 1039 (2004).

6. J.G. Tobin, G.D. Waddill, T.H. Gouder, C.A. Colmenares, and D.P. Pappas. MRS Symp. Proc. 313, 619 (1993).

7. J.L. Dehmer, A.F. Starace, and U. Fano, Phys. Rev. Lett. 26, 1521 (1971).

8. K.T. Moore, G. van der Laan, R.G. Haire, M.A. Wall, and A.J. Schwartz, Phys. Rev. B 73, 033109 (2006).

9. J.G. Tobin, B.W. Chung, R. K. Schulze, J. Terry, J. D. Farr, D. K. Shuh, K. Heinzelman, E. Rotenberg, G.D. Waddill, and G. Van der Laan, Phys. Rev. B 68, 155109 (2003) and references therein.

10. S. Heathman, R.G. Haire, T. Le Bihan, A. Lindbaum, M. Idiri, P. Normile, S. Li, R. Ahuja, B. Johansson, and G.H. Lander, Science 309, 5731 (2005); S. Heathman, R.G. Haire, T. Le Bihan, A. Lindbaum, K. Litfin, Y. Méresse, and H. Libotte, Phys. Rev. Lett. 85, 2961 (2000).

11. G. van der Laan and B.T. Thole, Phys. Rev. B 53, 14458 (1996). 